\title{
Gripping by controllable wet adhesion using a magnetorheological fluid
}

\author{
Michele Lanzetta ${ }^{a}$ (2), Karl Iagnemmab \\ ${ }^{a}$ Department of Civil and Industrial Engineering, University of Pisa, Italy \\ ${ }^{b}$ Laboratory for Manufacturing and Productivity, Massachusetts Institute of Technology, USA
}

The magnetorheological properties of ferrofluids (or smart, or active fluids) are well known, and are currently exploited in shear in advanced damping systems in the automotive industry, robotics (prosthesis), and machine tools (chatter reduction, positioning). This paper proposes an end effector for gripping by a novel form of controllable wet adhesion inspired by gastropod pedal mucus. The design of a gripper has been proposed, along with performance analysis based on experiments on various parameters, materials and surfaces, exhibiting robustness in unknown and dirty environment, typical of disassembly. Benefits over competing handling technologies and future research directions in this new area have been addressed.

handling; model; adhesion

\section{Introduction}

The reduction of lot sizes and increase in product variety [1, 2]for global competition and manufacturing efficiency makes automated assembly and disassembly [3] more demanding and challenging. Flexible automation to face decreasing manpower cost and more complex assembly tasks require the development of performing handling methods and devices.

Various principles have been inspired by nature to allow for gripping and adhesion [4]. Advances are recorded in dry adhesion inspired by certain lizards (geckos) and spiders [5] and in grasping by spines as in insects [6]. A novel form of controllable wet adhesion has been recently observed, although not completely understood, on gastropod (snails and slugs) pedal mucus [7].

As opposed to dry adhesion, where Van der Waals forces require large areas of intimate contact between the gripper's compliant structures and the surfaces to which they attach in order to achieve sufficient force, in wet adhesion, stronger forces can be achieved by interposing a glue, such as in pressuresensitive adhesives (PSA) like tape.

Among the main criticalities in wet adhesion are

- the relatively high forces for attachment and detachment,

- residue can remain on the handled part, and

- they are subject to rapid fouling by dust and dirt, decreasing performance over time.

In this paper the use a magnetorheological fluid as the medium in order to achieve controllable adhesion by a magnetic field and overcome the mentioned drawbacks of wet adhesion is proposed. By changing the magnetic field, not only attachment and detachment can be controlled, but also the adhesion strength.

It will be experimentally shown that this novel form of controllable wet adhesion can be applied to a wide range of surface conditions, i.e. substrate types and roughnesses (as opposed to suction), it can yield large clamping pressures without needing a ferrous substrate (as opposed to magnetic gripping) and potentially overcome problems with dust and other surface contaminants, e.g. oil from manufacturing operations, which are common in other types of dry and wet adhesion and suction. One potential drawback to consider when selecting the application is that the fluid deposited may stain the substrate with oil, although it has been observed that most of the fluid can be recovered.

\section{MR fluid (MRF) properties}

Magnetorheological fluids (MRFs) typically consist of a suspension of non-colloidal ferromagnetic particles in an inert oil. The selected MRF [8] is composed of iron particles between 1 and $20 \mu \mathrm{m}, 80 \%$ by weight, in synthetic hydrocarbon base oil. (NonNewtonian) fluids that change their viscosity by an electrical or magnetic field or similar are called active or smart fluids.

The magnetorheological properties of ferrofluids are well known. In engineering most literature on ferrofluids is on advanced damping systems and brakes in the automotive industry, in addition to machine tools (chatter reduction [9], positioning by controlled buoyancy [10] and polishing [11]) and robotics [12] (prostheses [13]).
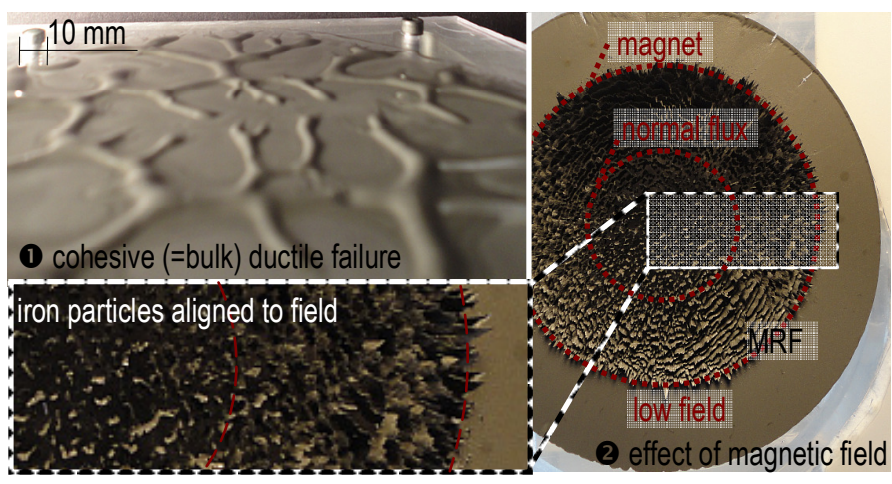

Figure 1. Effect of the magnetic field on the MR fluid 


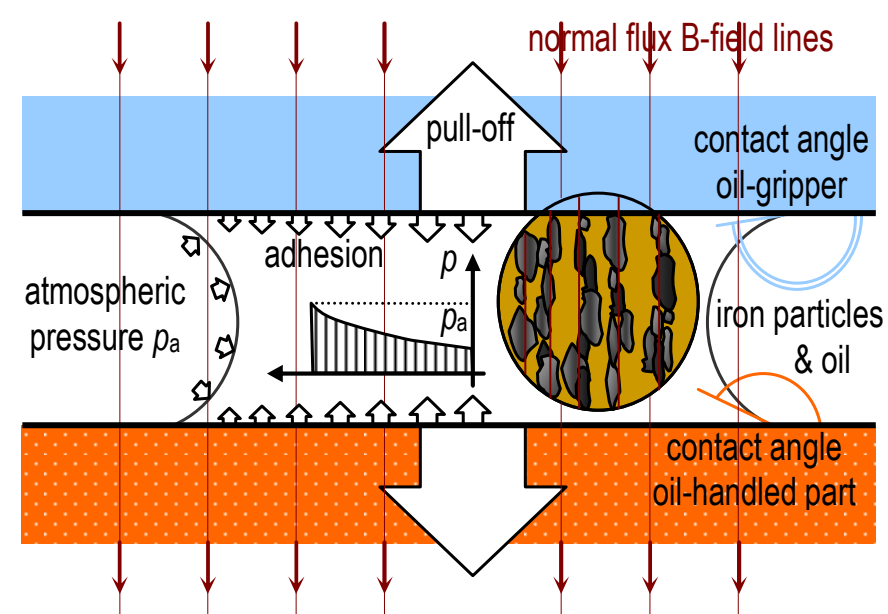

Figure 2. The proposed adhesion model

An external magnetic field induces magnetic dipoles in the particles, causing them to form chains along field lines. Figure 1.2 shows the alignment of particles, more and more inclined towards the edge (zone of influence) of the cylindrical magnet and flat outside, where the field is negligible.

This field-aligned anisotropic configuration strongly resists shear deformation to the viscosity increase with displacements perpendicular to the field lines.Most studies and applications subject MR fluids to shear loading (e.g. dampers). The proposed use of such fluids in this paper is as adhesives with the mechanism described in the next section.

MR fluid adhesive strength can be varied and controlled by varying the external magnetic field over several orders of magnitude. The yield stress on the MR fluid increases with the square of the applied magnetic flux $|\mathrm{B}|$ [7] and the maximum, which is material-specific, can be achieved at $0.6-0.9 \mathrm{~T}$ [8].

\section{Adhesion model}

Adhesion forces represented in Figure 2 are generated as a reaction to the pull-off force applied to the two plates. During

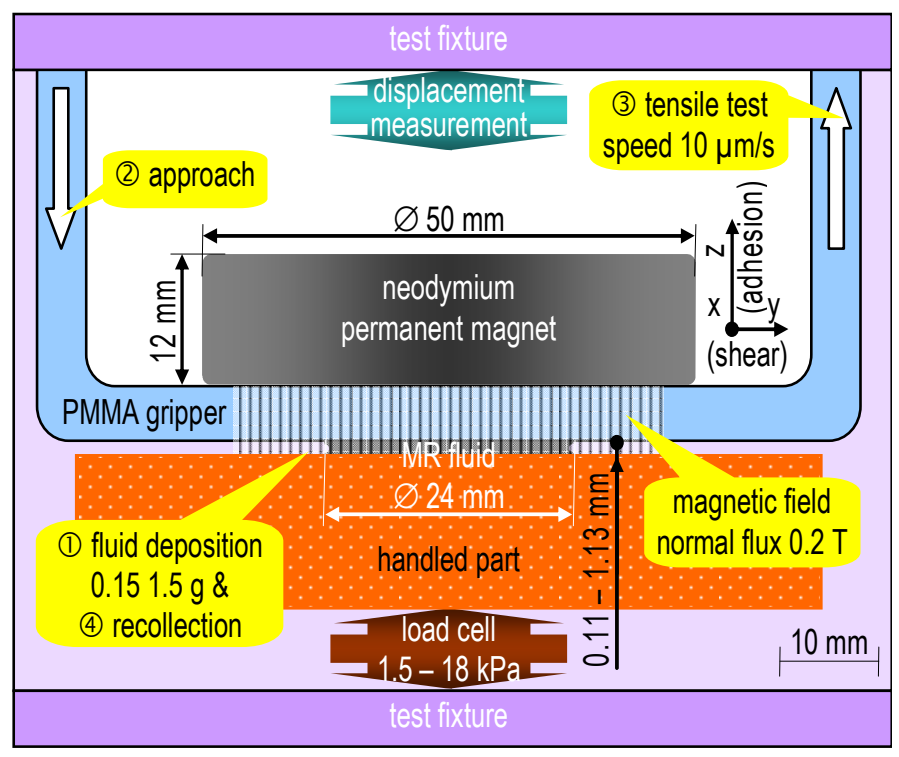

Figure 4. Conceptual scheme of an MR fluid [8] based gripper and working principle of the testing apparatus (actual dimension are shown)

separation, in order to keep its volume constant, the incompressible fluid is driven toward the center by the atmospheric pressure and the internal molecular cohesion forces. The negative pressure gradient produces adhesion between the fluid and the two solid surfaces.

This component of the adhesion force is additional to (molecular or Van der Waals) wetting or capillary forces, due to the difference in surface energy between oil and respectively the gripper and the part materials. In general the MRF shape after spreading is a truncated cone because of different wetting surfaces.

The MRF can be seen as a single use, easily removable temporary glue. The glue itself in turn can be considered like a specialized material, where the iron particles are an elastic metal structure supporting a compliant gripping material, the high viscosity (plastic) oil.

The grasping force is spread over the entire surface of the

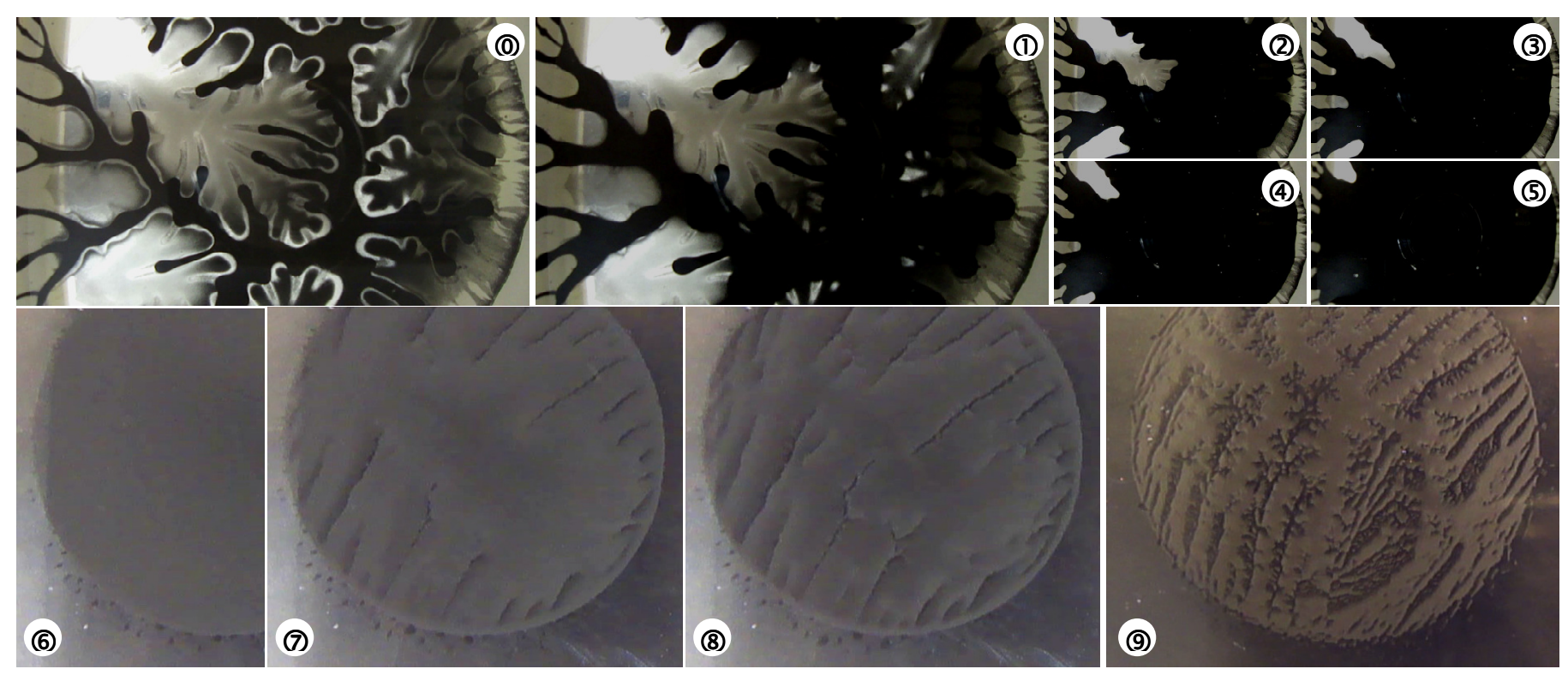

Figure 3. (0): video frame taken through a PMMA plate immediately after interfacial failure, showing the typical fjord shape with capillary adhesion. (1) to (5): reversible attachment sequence - similar to detachment - showing that fluid is reusable. (6) to (8): cohesive failure sequence, maximum strength 675 g, fluid thickness $0.51 \mathrm{~mm}$. (9): mixed case, interfacial failure (fjords) triggered from cracks, strength $780 \mathrm{~g}$, thickness $0.24 \mathrm{~mm}$ 


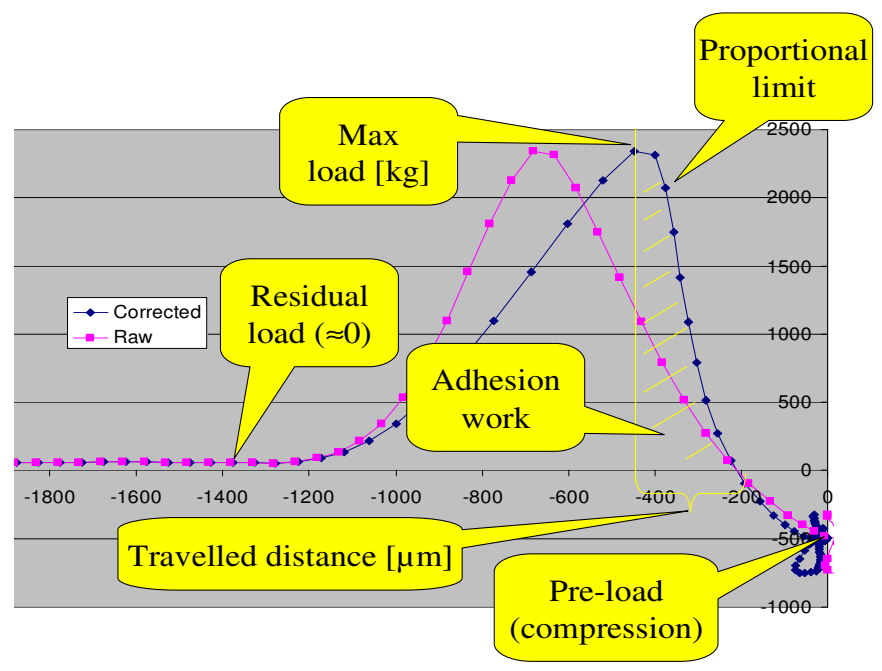

Figure 5. A typical force-displacement graph measured during tests with fixture stiffness correction in the experimental conditions of Figure 4 Load-displacement data are sampled at $200 \mathrm{~Hz}$

handled part thus reducing likelihood of damage.

In cohesive failure, the MR fluid yields and deforms in the bulk to relieve the applied strain. In a preliminary set of experiments it has been shown that cohesive failure occurs only at low magnetic fields, below approximately $0.1 \mathrm{~T}$ or when link chains (shown in the magnified circular inset of Figure 2 in actual colors) are degraded by repeated adhesion, as demonstrated in Figure 1.0, where the handled part with ripples of fluid is shown after separation from the gripper.

Activated MR fluid exhibits much higher adhesion than the ambient MR fluid $(|\mathrm{B}|=0 \mathrm{~T})$, making adhesion controllable as desired. At magnetic fields above $0.1 \mathrm{~T}$, the primary observed failure mechanism is interfacial; the MR fluid slowly detaches from the surface until failing in a single brittle event. This critical yield stress is of paramount engineering importance; it sets the boundary between adhesive and cohesive failure defining the maximum stress that can be obtained from a given sample of MR fluid.

In Figure 3 the two failure mechanisms are visually compared with a cylindrical $\varnothing 24 \mathrm{~mm}$ magnetic field. In cohesive failure, cracks propagate in the bulk and become barely visible at the interface as minute fragmentation. With lower fluid thickness, increased fragmentation has been observed; probably for the contribution of interfacial failure with less available volume of fluid. First cracks appear when the proportional limit in Figure 5 is reached.

\section{Gripper design}

This section describes the gripper design aspects involved with the innovative adhesion principle investigated. A scheme of the proposed gripper is shown in Figure 4. Experiments consider the basic configuration with parallel plates and normal adhesion.

Permanent magnets with locking mechanism to control the field are an option versus electromagnets where power saving is concerned, like in climbing robots. In industrial applications electromagnets seem more practical for the continuous control of the magnetic field by the electric current.

The gripper material should provide the maximum adhesion (obtained from both contributions: capillary force and MR adhesion); PMMA has been used in experiments to provide a direct view of the fluid (as in Figure 1 and Figure 3).

A known weight of fluid is deposited and the actual thickness is estimated from the measured diameter and determines the adhesion surface in the actual application.
A known issue is powder clumping and sedimentation and the consequent concentration change. This problem is being reduced in modern fluids by particle size and coating (e.g. by the patented surfactant decanedioic acid [8]).

Fluid recollection. Metal particles after separation of plates can be collected by a magnet leaving mainly oil residues on the handled part.

\section{Experiments}

Tests have been carried out using linear load-displacement machine TA.XTplus from Texture Technologies with the working principle in Figure 4 . A $\varnothing 50 \mathrm{~mm}, 12 \mathrm{~mm}$ thick neodymium disk (permanent) magnet has been used as the magnetic field source. An amount of MRF between 0.15 and $1.5 \mathrm{~g}$ in order to achieve the thickness range of 0.11 to $1.13 \mathrm{~mm}$ (in Figure 6 and Figure 7) at a nominal density of $3 \mathrm{~g} / \mathrm{cm}^{3}$ is deposited and then spread in order to achieve $\varnothing 24 \mathrm{~mm}$. This size is sufficient to overcome edge effects from surface tension and sufficiently smaller than that of the magnet to allow a homogeneous normal flux through the gripper plate of thickness $10 \mathrm{~mm} ;|\mathrm{B}|=0.2 \mathrm{~T}$, is high enough to achieve interfacial failure and not unnecessarily higher because failure occurs at the interface anyway.

An example of load-displacement graph is shown in Figure 5. It can be noticed that the fluid thickness increase caused by the fluid deformation under tensile stress is in the range 100-400 $\mu \mathrm{m}$ for all materials tested, with a mode of $115 \mu \mathrm{m}$. The corresponding diameter reduction is in the order of $4-8 \mathrm{~mm}$ depending on the initial thickness.

The preload (compression) is the force to achieve sufficient contact surface by having both surfaces wet by the MRF. After preload, which has the function to uniformly spread the fluid, eliminate bubbles etc., linearity starts. The MRF spreads easily because it is oil-based and dry solids that cannot be wet by hydrocarbon oil (oleophobic) are very rare. To spread the fluid and achieve the designed contact area $(\varnothing 24 \mathrm{~mm})$, the measured preload is in the range of $1-2 \mathrm{kPa}$.

The brittle failure effect shown by the sudden inclination change can be clearly observed.

The maximum measured load on different materials and surface roughnesses is shown in Figure 6.

Failure stresses are in the range of $\pm 11 \%$ of the maximum corresponding to 1 standard deviation. In decreasing importance, the main sources of experimental variability are: combinations of mechanisms of adhesion, MRF concentration, contact surfacedeposited amount. Considering the irregular trend of data, the effect of thickness on adhesion cannot be quantitatively assessed and apparently a lower thickness (and amount of fluid) is to be preferred.

Inversely to maximum load, from Figure 7 a positive correlation between time to failure and fluid thickness is observed at various loads tested. Safer grips are available at $20-40 \%$ of the maximum load. Consequently a larger amount of fluid would be beneficial for longer gripping time (over a minute).

Gripping a $522 \mathrm{~g}$ smooth aluminum disk using 0.3 to $1.5 \mathrm{~g}$ of fluid has shown an almost linear trend with failures at 13 to $28 \mathrm{~s}$ and thicknesses from 224 to $971 \mu \mathrm{m}$.

Another useful result relates to gripping speed, which has shown a positive correlation up to $5 \mathrm{~mm} / \mathrm{s}$, more than tripling the maximum load obtained at $10 \mu \mathrm{m} / \mathrm{s}$ of previously presented tests. $90 \%$ of the maximum load is still available in the speed range 1$20 \mathrm{~mm} / \mathrm{s}$. The positive effect of speed seems a consequence of shear stress being proportional to shear rate [8].

Combined shear and/or torque have been shown to be detrimental for normal pull-off force by preliminary tests with asymmetric loads, probably because the bridges are broken by a 


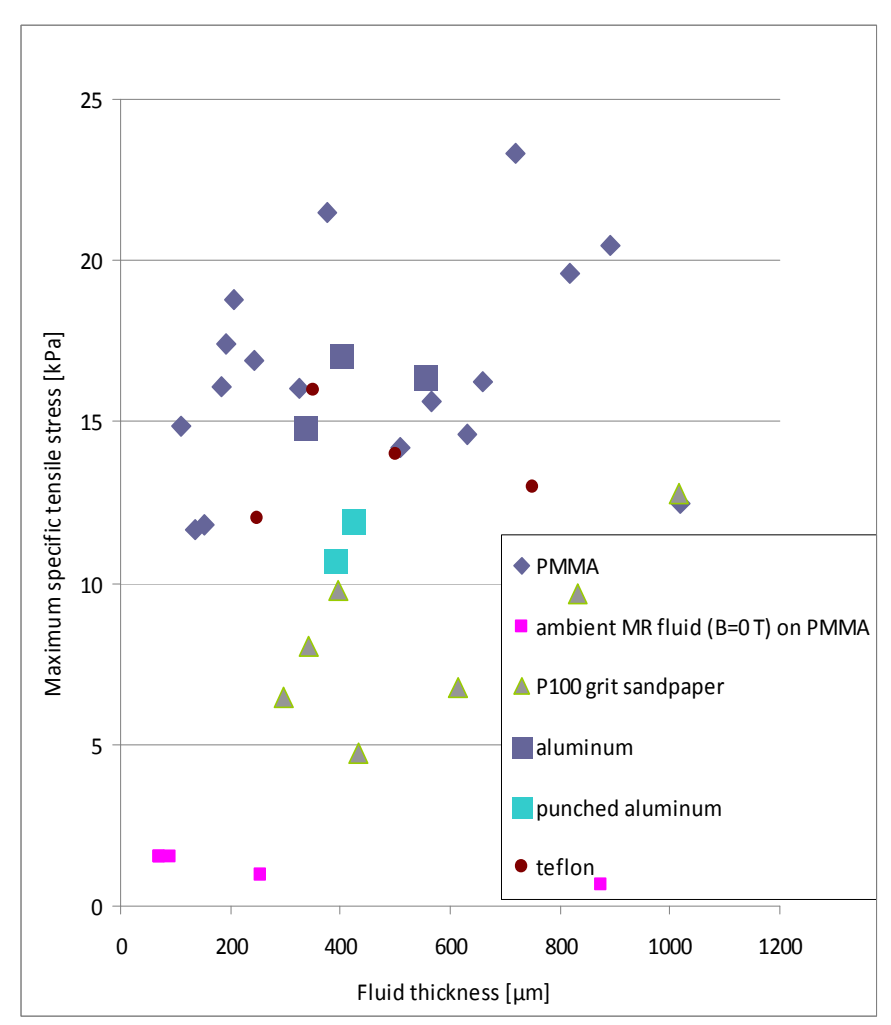

Figure 6. Adhesion on various materials and roughnesses with the experimental conditions in Figure 4 . Roughness values $R_{q}[\mu \mathrm{m}]$ : PMMA (0.1), smooth aluminum (0.2), rough aluminum (2) with punched holes, sandpaper (25), Teflon (0.2)

transverse relative movement and iron particles do not show rearrangement ability, when activated.

\section{Application}

Accurate part positioning. Load-displacement graphs (like the one in Figure 5) allow estimating the MRF deformation with load.

A limit of proportionality has been observed in most experiments around $85-92 \%$ of the maximum load and can be exploited for failure prediction.

No significant hysteresis has been observed by the fluid when reducing and increasing the load below this limit, showing that this fluid is elastic when activated (according to the general nonNewtonian fluid model).

Part release. If deactivated before failure the fluid still exerts a force of about $40 \%$ of the maximum, probably because of the ordered structure and the pressure gradient generated. This requires some disengage mechanism. A possibility is disengaging by a transverse movement, like flexion, shear, torque, peeling or their combinations to break the chains followed by field reactivation to pick up MRF debris. Some preliminary shear tests have shown lower performance compared to tensile tests (in the order of $7 \mathrm{kPa}$ for sandpaper), as opposed to common adhesives.

Fluid degradation with use can be considered negligible, because life in dampers is in the order of years and million of cycles and single use is the most probable exploitation (unless differently addressed by future work).

Recollection. Because the oil wets well iron particles, if they are recollected by a magnet, part of the oil will also be recollected.

Contamination. After interfacial failure, most of the fluid, particularly iron particles, remain on the gripper surface, which is closer to the magnet. The measured amount of fluid lost, mostly oil, is less than $10 \%$, however the fluid cannot be reused as is

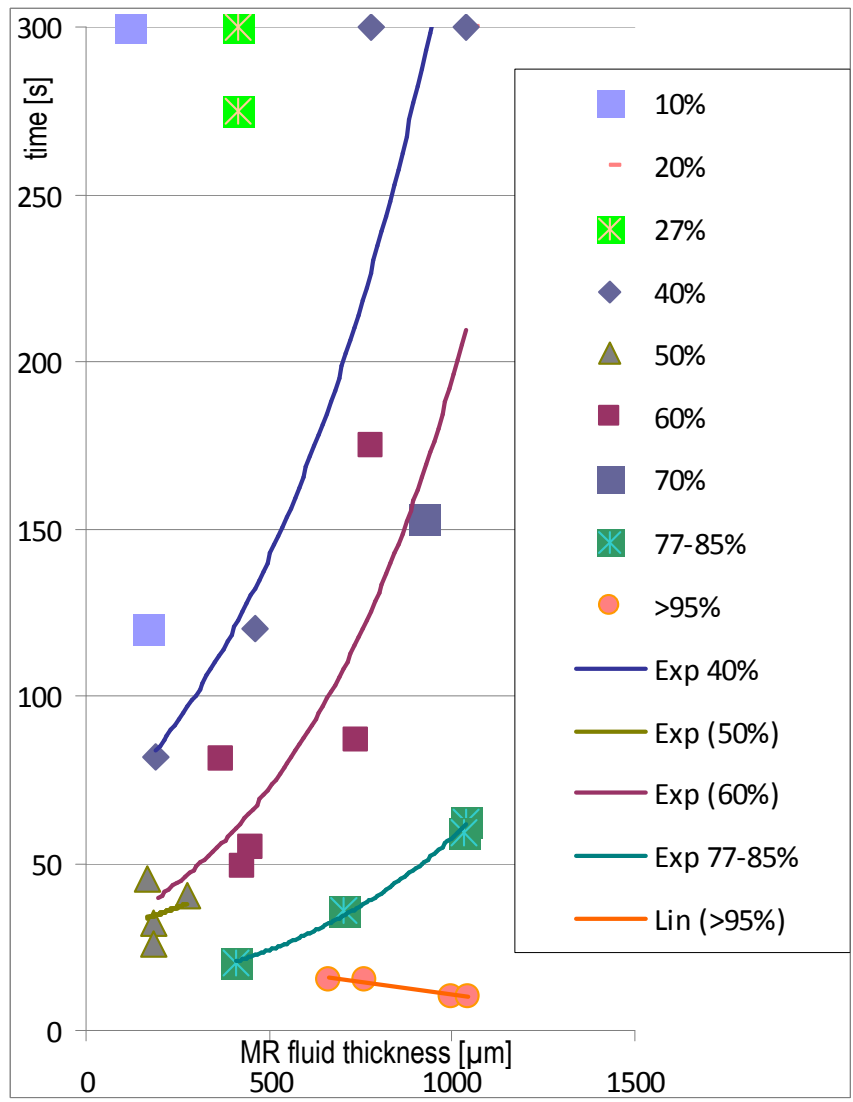

Figure 7. Time to failure (test time limited to $300 \mathrm{~s}$ ) at different normal stress levels (top), with the experimental conditions in Figure 4 on PMMA as the handled material

because of the concentration change. Hydrocarbon oils can harm some plastics like natural rubber, butyl, EPDM/EPR and silicone, but different oils can be used.

\section{Benchmarking}

In addition to the benefits mentioned in the introduction, regarding the main competing technologies, as opposed to vacuum, the noise is negligible and there is no need of flexible piping; the effect of roughness has been shown to be beneficial, because it increases shear, although reducing adhesion. In addition, complex shapes or the presence of holes or porous materials, where vacuum fails, has the only effect of reducing adhesion proportionally to the reduced contact surface. Holes do not seem fracture triggers, because interfacial failure is of brittle type.

As opposed to magnetic grippers, MR fluids can be used for switchable normal force adhesion to non-magnetic substrates. A major benefit is that they work with non ferromagnetic metals, like aluminum, gold, silver, and copper, in addition to plastics. With permanent magnets there is no need of power line.

Some orders of magnitude are also provided. The specific magnetic force on an equivalent surface, given $|\mathrm{B}|=0.2 \mathrm{~T}$ has the following theoretical maximum (Maxwell's equation with air permeability $\mu_{0}$ ) for a ferromagnetic handled part, $F_{\text {magnet }}=\frac{B^{2}}{2 \mu_{0}}$ $\cong 16 \mathrm{kPa}$; for a suction cup in atmospheric pressure, $\mathrm{F}_{\text {suction }}=101$ $\mathrm{kPa}$; from Figure $6, \mathrm{~F}_{\text {adhesion }}=\mathrm{F}_{\text {capillary }}+\mathrm{F}_{\mathrm{MRF}} \cong 20 \mathrm{kPa}$.

A synergy between a magnetic gripper where already available and the MRF-based gripper is also proposed. The dispensed MRF 
will increase load and extend action on non-ferromagnetic materials.

The variety of tested materials, from PMMA to aluminum and Teflon (which is incompatible to most polymeric glue) and roughnesses, from sandpaper $\left(R_{q}=25 \mu \mathrm{m}\right)$ to rough $\left(R_{q}=2 \mu \mathrm{m}\right)$ and smooth aluminum $\left(R_{\mathrm{q}}=0.2 \mu \mathrm{m}\right)$, have shown a performance variation within $\pm 15 \%$. In comparison to other adhesive technologies, MR fluid adhesion may be of utility with unknown, dirty, or varied product surfaces and materials, typical of disassembly environments.

\section{Conclusion}

The proposed gripper can reliably hold for over one minute with approximately $5 \mathrm{kPa}$, given a deposit of $0.8 \mathrm{~g}$ of MRF, resulting in a nominal cost of 0.3 US\$ per handled part, and a maximum normal load of $40 \mathrm{kPa}$ (not shown in graphs). The high cost issue, in the order of 1 kUS\$ per liter, can be cut by three orders of magnitude using standard oil and ferroparticles in handling application, where the operative life of the fluid is only a few seconds and then wasted, and by including a stirring device in the fluid reservoir.

Numerous open questions remain about controllable wet adhesion, opening a new range of research opportunities spanning from gripper configuration (e.g. compliant grippers), to fluid dispensing and recollection and magnetic field control devices, for different materials (e.g. soft materials), shapes (e.g. curved or sharp) and loading combinations (e.g. normal force, shear, torque).

\section{Acknowledgements}

Thanks are due to: Nicholas Wiltsie and the staff of the Robotic Mobility Group of the Laboratory of Manufacutring and Productivity (MIT) for their many suggestions and assistance in experiments; Jung-Hoon Chun for allowing this project; Marco Santochi's advice on the first draft. Karl Iagnemma would like to acknowledge the support of Battelle for project funding.

\section{References}

[1] Hu SJ, Ko J, Weyand L, ElMaraghy HA, Lien TK, Koren Y, Bley H, Chryssolouris G, Nasr N, Shpitalni M (2011) Assembly system design and operations for product variety. CIRP Annals 60(2):715-733, doi:10.1016/i.cirp.2011.05.004.

[2] Daaboul J, Da Cunha C, Bernard A, Laroche F (2011) Design for mass customization: Product variety vs. process variety. CIRP Annals 60(1):169-174, doi:10.1016/j.cirp.2011.03.093.

[3] Hauschild M, Jeswiet J, Alting L (2005) From Life Cycle Assessment to Sustainable Production: Status and Perspectives, CIRP Annals 54(2):1-21, doi:10.1016/S0007-8506(07)60017-1.

[4] Shu LH, Ueda K, Chiu I, Cheong H (2011) Biologically inspired design. CIRP Annals 60(2):673-693, doi:10.1016/i.cirp.2011.06.001.

[5] Lanzetta M, Cutkosky MR (2008) Shape Deposition Manufacturing of Biologically Inspired Hierarchical Microstructures. CIRP Annals 57(1):231-234, doi:10.1016/i.cirp.2008.03.102.

[6] Asbeck AT, Kim S, Cutkosky MR, Provancher WR, Lanzetta M (2006) Scaling Hard Vertical Surfaces with Compliant Microspine Arrays. International Journal of Robotics Research 25(12):1165-1180, doi:10.1177/0278364906072511.

[7] Ewoldt RH, Tourkine P, McKinley GH, Hosoi AE (2011) Controllable adhesion using field-activated fluids. Physics of Fluids 23:073104.

[8] MRF-132DG Magneto-Rheological Fluid, LORD Technical Data, 1-2, 07/2008. http://www.lord.com.

[9] Mei D, Kong T, Shih AJ, Chen Z (2009) Magnetorheological fluid-controlled boring bar for chatter suppression. Journal of Materials Processing Technology 209(4):1861-1870, doi:10.1016/i.jmatprotec.2008.04.037.

[10] Uhlmann E, Bayat N (2006) High Precision Positioning with Ferrofluids as an Active Medium. CIRP Annals 55(1):415-418, doi:10.1016/S00078506(07)60448-X.

[11] Jang K-I, Seok J, Min B-K, Lee SJ (2010) An electrochemomechanical polishing process using magnetorheological fluid. International Journal of Machine Tools and Manufacture 50(10):869-881, doi:10.1016/j.ijmachtools.2010.06.004.

[12] Wiltsie N, Lanzetta M, Iagnemma K (2012) A Controllably Adhesive Climbing Robot Using Magnetorheological Fluid, 4th IEEE International Conference
TePRA, Woburn, MA,
doi:10.1109/TePRA.2012.6215660.

USA,

23-24/04/2012:91-96,

3] Carlson JD, Matthis W, Toscano JR (2001) Smart prosthetics based on magnetorheological fluids. Proceedings of SPIE 4332:308-316. 